\title{
Preface Issue 1-2015
}

\author{
Hans-Christoph Grunau ${ }^{1}$
}

(C) Deutsche Mathematiker-Vereinigung and Springer-Verlag Berlin Heidelberg 2015

A typical example from optimal control with partial differential equations is as follows. Consider a domain where one wants to achieve a desired temperature distribution $y_{d}$. For this purpose one may use heat sources $u$ which, at a given time, will result in a real temperature distribution $y_{u}$. In order to compute $y_{u}$ from $u$ one has to solve a typically nonlinear heat equation. In most cases $y_{d}$ and $y_{u}$ will be different. The goal will be to find a control $u$ such that $y_{d}-y_{u}$ becomes as small as possible in a suitable sense while at the same time the costs for the heating $u$ stay as low as possible. Both requirements are combined in a functional $u \mapsto J(u)$ and the task is to decide whether a given control $u$ minimises $J$. As in first year calculus, one will have to take a look at the equation $J^{\prime}(u)=0$. Unless $J$ is convex it will be very difficult to detect global minima, but one would also be glad to find local minima. To this end, one will have to check the positive definiteness of $J^{\prime \prime}(u)$. Unlike first year calculus, the space of admissible controls is now infinite dimensional, and one has to consider different norms in order to ensure differentiability and positive definiteness. Starting with this observation, Eduardo Casas and Fredi Tröltzsch explain in a very comprehensible way "Second order optimality conditions and their role in PDE control."

Thanks to the increasing demand of renewable energies it has become an important scientific question how to safely exploit geothermal energy. Geothermal engineering relies in many respects on the mathematical modelling and analysis and the efficient design and implementation of numerical methods. Willi Freeden and Helga Nutz's survey article explains how mathematics is a key technology in understanding deep

H.-Ch. Grunau

hans-christoph.grunau@ovgu.de

1 Institut für Analysis und Numerik, Fakultät für Mathematik, Otto-von-Guericke-Universität, Postfach 4120, 39016 Magdeburg, Germany 
geothermal systems. The article addresses in particular the questions of how to detect geothermal reservoirs, how to model and simulate numerically the related heat flows and the changes of mechanical tensions. With regard to the last problem, one should have in mind seismic events as in Staufen (2008) or light earth tremors as e.g. in Basel (2006) or in St. Gallen (2013).

In order to compensate for so much applied analysis and numerics, Bernhard Mühlherr gives a detailed review of the book on "Diagram geometry", written by Francis Buekenhout and Arjeh M. Cohen. 